Copyright by the Acoustical Society of America. Beyene, S., \& Burdisso, R. A. (1997). A new hybrid passive/active noise absorption system. Journal of the

Acoustical Society of America, 101(3), 1512-1515. doi: 10.1121/1.418105

\title{
A new hybrid passive/active noise absorption system
}

\author{
Samson Beyene and Ricardo A. Burdisso \\ Vibration and Acoustics Laboratories, Mechanical Engineering Department, Virginia Polytechnic Institute \\ and State University, Blacksburg, Virginia 24061-0238
}

(Received 14 August 1995; revised 6 May 1996; accepted 15 October 1996)

\begin{abstract}
A new hybrid passive/active system is developed for sound absorption over a wide frequency range. The system is comprised of a layer of absorbing material positioned at a distance from an active wall, leaving an air space. The motion of the active wall is based on a new control approach which consists of the minimization of the reflected wave within the airspace which modifies the layer's back surface impedance so as to match the characteristic impedance of air. This technique is referred here as inducing an impedance-matching condition. Both numerical and experimental results of such a system are presented for normally incident planar waves. The hybrid passive/active system results in a high absorption coefficient of .8-1.0 over the frequency range 100-2000 Hz and is insensitive to system parameters such as air space depth and absorbing layer thickness. (C) 1997 Acoustical Society of America. [S0001-4966(97)02303-5]

PACS numbers: 43.55.Ev, 43.50.Ki [PJR]
\end{abstract}

\section{INTRODUCTION}

In general, passive noise control methods that employ the use of sound-absorbing materials are practical and most effective at mid to high frequencies. On the other hand, active noise control techniques are more efficient at low frequencies. The complementary strengths and weaknesses of passive and active noise control methods have motivated many researchers to develop a system that integrates both methods. One of the first published works on a noise absorption system that comprises both active and passive control methods is that of Guicking and Lorenz. ${ }^{1}$ The passive component was comprised of a porous plate located in an impedance tube a small distance from the open end of the tube which was terminated by a control speaker. The signal from a microphone in front of the porous plate was sent to the control speaker after it was passed through a suitable amplification scheme. A second microphone controlled the complex amplification factor such that the sound pressure at that location was minimized so as to produce a pressure-release condition just behind the plate. Almost total absorption of the acoustic energy was reported over the frequency range 100-600 Hz.

Thenail et al. ${ }^{2}$ investigated an active system that included a fiberglass absorbing layer backed by an air cavity terminated with an active surface. Their work was intended to show that a pressure-release condition on the back surface of the fiberglass leads to improvement in absorption. They investigated two different control approaches. In the first control approach, an error microphone was located on the back surface of the fiberglass layer, and the pressure at that location was minimized. The result of this study showed an improvement of absorption that declines with an increase in frequency. The result reported was for the frequency range of 200-800 Hz. The second control approach investigated was identical to Guicking and Lorenz's work, ${ }^{1}$ where the porous plate was replaced with the fiberglass layer. Their results show almost total absorption for the frequency range of 500$1400 \mathrm{~Hz}$. In both control approaches, the authors used an optimum fiberglass layer thickness of $2 \mathrm{~cm}$. Both the numerical and experimental results demonstrated that the system was sensitive to fiberglass thickness and porosity.

Fuller et $a .^{3}{ }^{3}$ presented an experimental study done on the potential of an "adaptive foam'" for radiation and reflection control. Their adaptive foam included a polyvinylidene fluoride film (PVDF) imbedded in polyurethane foam in a sine wave shape. In the reflection control experiment, the adaptive foam was rigidly backed and positioned at the open end of an impedance tube. The incident and reflected waves in front of the adaptive foam were separated using a set of identical microphones and a wave deconvolution circuit, and the reflected wave signal was minimized. The experiment performed was for a frequency range of 100-1000 Hz. At frequencies above $600 \mathrm{~Hz}$, attenuation of the reflected wave of up to $40 \mathrm{~dB}$ was reported. Without active control, the passive attenuation of the adaptive foam was reported to be low.

A hybrid passive/active system for sound absorption which is again based on the concept of mounting a layer of sound-absorbing material at a distance from a wall is proposed here. Unlike in previous work where the wall is driven so as to produce a pressure-release condition at the back surface of the layer, ${ }^{1,2}$ in this new approach the impedance at the back surface of the layer is modified to match the characteristic impedance of air. This condition is referred here as impedance matching to differentiate it from the pressurerelease (or zero impedance) condition. In practice, the impedance-matching condition is simply achieved by minimizing the reflected wave in the airspace behind the absorbing layer. The effectiveness of this approach is demonstrated both numerically and experimentally for normal incident waves and single frequency excitations.

\section{CONTROL APPROACH}

A simple one-dimensional (1-D) numerical model is developed to simulate the proposed hybrid passive/active system. This system was modeled as a two-layer media where 


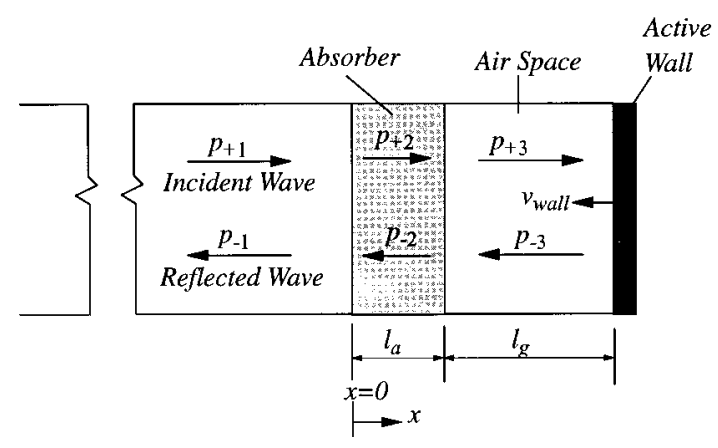

FIG. 1. Passive/active sound absorption system.

the first medium is a layer of a sound-absorbing material and the second is a cavity of air, backed by an active wall as shown in Fig. 1. The fluid in front of the absorbing layer is also assumed to be air. The incident and reflected waves in each of the three regions are represented in their exponential form as

$$
\begin{gathered}
p_{+n}(x, t)=A_{n} e^{-\Gamma x} e^{i \omega t}, \quad p_{-n}(x, t)=B_{n} e^{\Gamma x} e^{i \omega t}, \\
n=1,2,3
\end{gathered}
$$

where the coefficients $A_{n}$ and $B_{n}$ are the amplitude of the incident and reflected waves, respectively; $\omega=2 \pi f$ is the angular frequency; $\Gamma$ is the propagation constant; and $i$ $=\sqrt{-1}$. Due to the dissipative nature of the absorbing material $(n=1)$, the propagation constant $\Gamma$ is complex and can be expressed as $\Gamma_{a}=\xi_{a}+i k_{a}$, where the real part $\xi_{a}$ is the attenuation constant and the imaginary part $k_{a}$ is the phase constant. In the airspace and in front of the absorber, the propagation constant is purely imaginary and equal to the acoustic wave number given as $i k=i \omega / c$, where $c$ is the speed of sound in air.

The particle velocity for the above waves can be written in terms of the plane wave impedance of the medium as

$$
\begin{gathered}
\nu_{ \pm 2}(x, t)= \pm \frac{p_{ \pm 1}(x, t)}{Z_{a}}, \quad \nu_{ \pm n}(x, t)= \pm \frac{p_{ \pm n}(x, t)}{\rho c} \\
n=1,3
\end{gathered}
$$

where $Z_{a}$ is the complex impedance of the absorbing material and $\rho c$ is the characteristic impedance of air.

The conditions of continuity of pressure and particle velocity are now imposed at the front and back surfaces, e.g., $x=0$ and $x=l_{a}$, of the absorbing layer as well as the matching of the fluid particle and active wall velocities at $x=l_{a}+l_{g_{3}}$. These conditions lead to the linear system of equations $^{3}$

$$
\left[\begin{array}{ccccc}
-1 & 1 & 1 & 0 & 0 \\
\hat{Z}_{a} & 1 & -1 & 0 & 0 \\
0 & E^{-1} & E & -D^{-1} & -D \\
0 & E^{-1} & -E & -D^{-1} \hat{Z}_{a} & D \hat{Z}_{a} \\
0 & 0 & 0 & G^{-1} & -G
\end{array}\right]\left\{\begin{array}{c}
B_{1} \\
A_{2} \\
B_{2} \\
A_{3} \\
B_{3}
\end{array}\right\}
$$

$$
=\left\{\begin{array}{c}
1 \\
\hat{Z}_{a} \\
0 \\
0 \\
0
\end{array}\right\} A_{1}+\left\{\begin{array}{c}
0 \\
0 \\
0 \\
0 \\
\rho c
\end{array}\right\} \nu_{\text {wall }}
$$

where $E=\exp \left(\Gamma_{a} l_{a}\right), D=\exp \left(i k l_{a}\right), G=\exp \left(i k\left(l_{a}+l_{g}\right)\right)$, and $\hat{Z}_{a}=Z_{a} / \rho c$.

The optimum control input $\left(\nu_{\text {wall }}\right)_{\text {opt }}$ should be determined to minimize the total reflected wave, $B_{1}$, in front of the absorbing layer. One approach is the use of a wave sensor that directly measures the total reflected wave, $B_{1}$. This sensing approach was implemented previously in the work by Fuller et al. $^{4}$ and Guicking and Karcher ${ }^{5}$ in purely active systems. However, it presents a design drawback since the error transducer is located in the acoustic field to be minimized, i.e., zone of quiet. This is impractical in many applications, a fact clearly recognized by Guicking and Karcher. ${ }^{5}$ Here, we seek a sensing strategy where, first, the error transducer is positioned in the airspace (so it does not interfere with the system, i.e., the error sensor is not in the zone of quiet) and, second, by canceling the measured acoustic response in the airspace, minimization of the total reflected wave in front of the system is obtained. To this end, it is important to review the behavior of a layer of absorbing material for normal incident waves.

The input impedance, $Z_{I}$, of a layer of absorbing material can be computed from the impedance at the back surface of the absorbing layer $\left(Z_{2}\right)$, the acoustical properties of the material $\left(\Gamma_{a}\right.$ and $\left.Z_{a}\right)$, and the layer thickness $\left(l_{a}\right)$ as $^{6}$

$$
Z_{I}=Z_{a} \frac{Z_{2} \cosh \left(\Gamma l_{a}\right)+Z_{a} \sinh \left(\Gamma l_{a}\right)}{Z_{a} \cosh \left(\Gamma l_{a}\right)+Z_{2} \sinh \left(\Gamma l_{a}\right)} .
$$

The impedance at the back surface of the absorbing layer can be computed from the relative values of the incident and reflected sound waves in the airspace as

$$
Z_{2}=\rho c \frac{A_{3} e^{-i k l_{a}}+B_{3} e^{i k l_{a}}}{A_{3} e^{-i k l_{a}-B_{3} e^{i k l_{a}}} .}
$$

Replacing the last equation in the linear system of Eq. (3) into (5) leads to

$$
Z_{2}=\rho c \frac{\rho c \nu_{\text {wall }}+B_{3} e^{i k l_{a} 2} \cos \left(k l_{g}\right)}{\rho c \nu_{\text {wall }}+B_{3} e^{i k l_{a}} \sin \left(k l_{g}\right)},
$$

where it is clear that the impedance at the back surface of the absorbing layer is a function of the active wall velocity, i.e., explicitly through the first terms in the numerator and denominator and implicitly through the wave amplitude $B_{3}$. Setting $\nu_{\text {wall }}$ to zero in Eq. (6) yields the well-known impedance $Z_{2}=-i \tan \left(k l_{g}\right)$ due to a rigid wall at a distance $l_{g}$ from the absorber layer. ${ }^{6}$

Equation (6) clearly indicates that the active control system, i.e., motion of the wall, can be interpreted as an active modification of the layer back surface impedance and consequently of the input impedance $Z_{I}$ as well. In this context, the control approach of minimizing the pressure at the back surface of the layer (referred as pressure release condition) is equivalent to imposing a zero impedance condition, $Z_{2}=0$. 


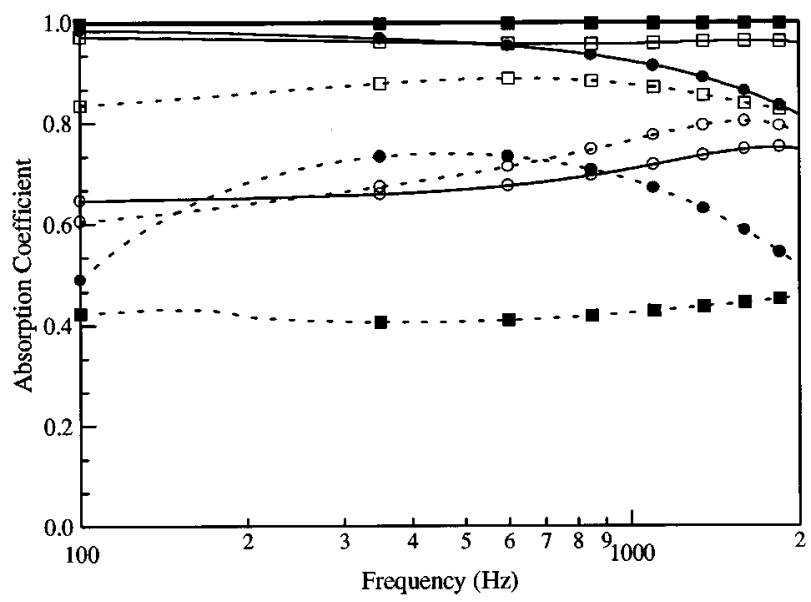

FIG. 2. Absorption coefficient of absorbing layer with (_- impedancematching and (---) pressure-release conditions at the layer's back surface: fiberglass, $\square$ foam, $\bigcirc$ sponge rubber, and $\bigcirc$ Kevlar.

The optimum impedance, $Z_{2}$, that would lead to perfect absorption can easily be obtained by setting the input impedance equal to the characteristic impedance of air, i.e., $Z_{I}=\rho c$ in Eq. (4), and solving for $Z_{2}$. This optimum impedance is a complex function of the acoustical properties and thickness of the absorbing layer and may be difficult to implement in practice. However, further inspection of Eq. (4) reveals that as the arguments of the hyperbolic functions decrease $\left(\Gamma l_{a} \rightarrow 0\right)$, the $\cosh \left(\Gamma l_{a}\right)$ and $\sinh \left(\Gamma l_{a}\right)$ function will approach unity and zero, respectively. This implies that the optimum back impedance should approach the characteristic impedance of air, i.e., $Z_{2}=\rho c$, in spite of the value of the characteristic impedance of the absorbing material, $Z_{a}$. The decrease in the argument $\Gamma l_{a}$ will take place when (a) the thickness of the absorbing layer is reduced and/or (b) when the propagation characteristic is reduced which occurs in most acoustic-absorbing materials as the frequency is decreased. Thus, an impedance equal to the characteristic impedance of air at the back surface of the absorbing layer is the optimum value for a thin layer and low frequencies. An impedance of $Z_{2}=\rho c$ is referred to here as impedancematching condition.

To further validate the above conclusions, the input absorption coefficient for various absorbing materials is computed assuming both impedance-matching and pressurerelease conditions at the back of the absorbing layer. The absorbing materials selected for this analysis are fiberglass, ${ }^{6}$ open cell foam, ${ }^{7}$ Kevlar, ${ }^{7}$ and sponge rubber, ${ }^{8}$ all of which have markedly different acoustic characteristics. Figure 2 shows the absorption coefficient as a function of the frequency for a layer thickness of $0.5 \mathrm{~cm}$. The results show that the impedance-matching condition leads to better absorption characteristics than the pressure-release condition at all frequencies except for Kevlar, where the absorption coefficient is essentially the same for both conditions. Figure 3 shows the absorption coefficient as a function of the absorbing layer thickness, $l_{a}$, for the various materials at a frequency of 300 $\mathrm{Hz}$. The results indicate that there is an optimum thickness, which differs for the different materials, to obtain high absorption when a pressure-release condition is induced. Thus,

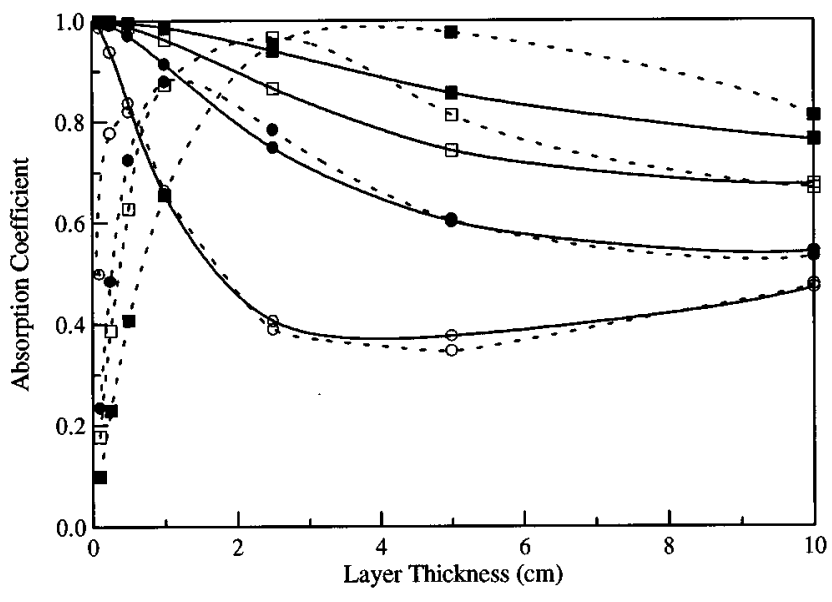

FIG. 3. Absorption coefficient at $300 \mathrm{~Hz}$ as a function of the absorbing layer thickness, $l_{a}$, for (- impedance-matching and (---) pressure-release conditions: $\square$ fiberglass, $\square$ foam, sponge rubber, and $\bigcirc$ Kevlar.

the performance of the sound-absorbing system with an actively induced pressure-release condition at the back of the absorbing layer is very sensitive to the thickness of the absorbing layer. This fact was also observed by Thenail et al. ${ }^{2}$ On the other hand, for the impedance-matching condition, absorption decreases slowly as the thickness, $l_{a}$, is increased, i.e., the system is insensitive to the absorbing layer thickness. Figure 3 also shows that the impedance-matching condition outperforms the pressure-release condition in most situations, in particular for thin layers.

Based on the previous analysis, a new active system for sound absorption is proposed which consists of introducing an impedance-matching condition at the absorbing layer back surface. This impedance-matching condition is easily achieved by the sensing and minimization of the reflected wave within the airspace. The minimization of the reflected wave in the airspace causes the acoustic impedance along the airspace (and at the back of the absorbing layer) to be equal to the characteristic impedance of air. In practice, the reflected wave in the airspace can be sensed by using a twomicrophone system. ${ }^{3,5}$

\section{NUMERICAL AND EXPERIMENTAL RESULTS}

The numerical model described above is used to investigate the performance of the proposed passive/active sound absorption system. The system investigated is comprised of a $5-\mathrm{cm}$ partially reticulated polyurethane foam layer and an airspace depth of $10 \mathrm{~cm}$. The density of air $\rho$ and the speed of sound $c$ in air are assumed to be $1.21 \mathrm{~N} . \mathrm{s}^{2} / \mathrm{m}^{4}$ and 343 $\mathrm{m} / \mathrm{s}$, respectively. The complex propagation constant, $\Gamma_{a}$, and characteristic impedance, $Z_{a}$, of the foam were empirically determined. ${ }^{4}$

The absorption coefficient of the passive system as a function of the frequency is shown in Fig. 4. The optimum active wall velocity is computed from Eq. (3) to minimize $B_{l}$ and is used to predict the absorption coefficient for the passive/active system also shown in Fig. 4. As the figure shows, the minimization of the reflected wave in the airspace successfully resulted in a high absorption coefficient over the whole frequency range of interest. Although the absorption 


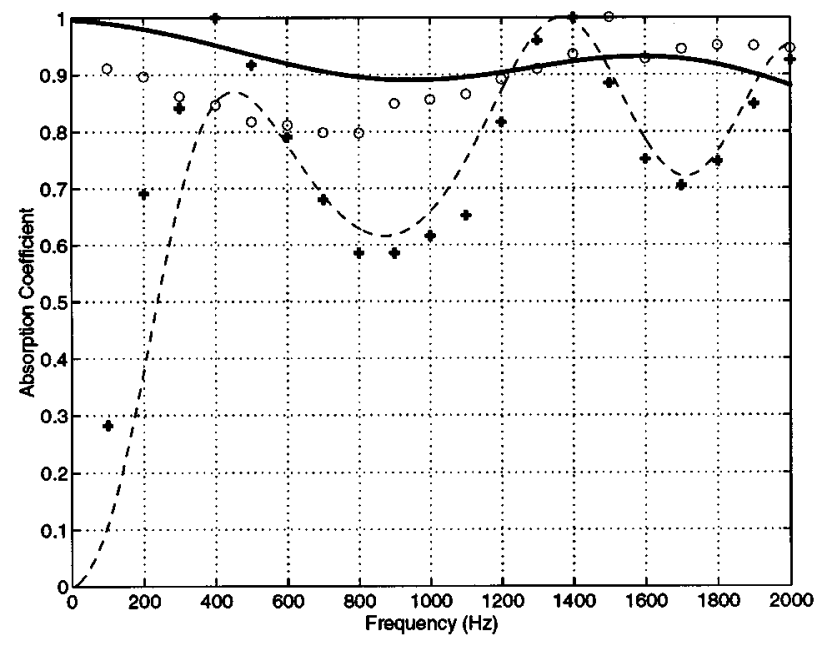

FIG. 4. Absorption coefficient of passive/active system: analytical, --- before and - after control; experimental, +++ before and $\bigcirc \bigcirc \bigcirc$ after control.

peaks at 1350 and $2000 \mathrm{~Hz}$ achieved by the passive system were slightly reduced with the introduction of the active component, overall, a consistent high absorption coefficient of 0.89-1.0 was achieved throughout the frequency range of interest. Moreover, the best performance of this hybrid system occurs at frequencies below the first peak of the passive system, i.e., $f<450 \mathrm{~Hz}$, where improvement is most desirable.

An experimental validation of the proposed passive/ active system was also performed. Due to the onedimensional nature of the model, the experiments performed were carried out in a standard impedance tube with a $10-\mathrm{cm}$ diameter and lower and upper frequency limits of 90 and $2000 \mathrm{~Hz}$, respectively. The disturbance source is located at one end of the impedance tube and the other end of the tube is terminated with a control speaker. The sound-absorbing layer, a 5-cm-thick partially reticulated foam, is placed at 10 $\mathrm{cm}$ from the control speaker, creating an airspace between the foam and the control speaker. The absorption coefficient of the system was measured using the standing wave technique (SWR). ${ }^{9}$ A wave deconvolution circuit first described by Fahy ${ }^{10}$ was used to sense the reflected wave in the airspace. This circuit gives a real time domain estimate of the incident and reflected components of a sound field from the outputs of two closely spaced identical microphones. The two microphones used in this experiment were located in the airspace with a 5-cm spacing between them with the closest microphone to the sound-absorbing layer at $1.25 \mathrm{~cm}$. The signals detected by these microphones were fed into the wave deconvolution circuit where the incident and reflected waves in the airspace were then separated and the reflected wave signal processed by a single-channel-filtered X-LMS controller as the error signal. ${ }^{11}$ The reference signal was simply tapped from the disturbance. The required control signal to minimize the reflected wave was then generated by the controller and sent to the control speaker. The sampling rate for each experiment was four times the operating frequency.

The experimental results for both before and after control cases are also presented in Fig. 4. As shown in the figure, a high absorption coefficient of $0.8-1.0$ is achieved experimentally over the frequency range $100-2000 \mathrm{~Hz}$. At the lower frequencies, the experimentally obtained absorption coefficient tends to be lower than the numerical result. The maximum deviation between the experimental and numerical results is an absorption coefficient difference of 0.1. This deviation occurs at the lower frequencies due to a phase mismatch between the two particular microphones used. In spite of this problem, the absorption coefficient at $100 \mathrm{~Hz}$ is increased from 0.28 to 0.91 . Overall, a good agreement between the numerical and the experimental results is observed which validates the proposed approach.

\section{CONCLUSIONS}

A new passive/active sound absorption system is proposed which consists of a layer of sound-absorbing material and an airspace terminated by an active wall. The motion of the wall is used to modify the layer's back impedance so as to match the characteristic impedance of air. This is accomplished by sensing and minimizing the reflected wave in the airspace. The numerical results show high absorption (0.81.0) over a wide frequency range of $100-2000 \mathrm{~Hz}$. The proposed system is insensitive to the absorbing layer thickness and airspace depth. An experimental study is also carried out in an standing wave tube. The very good agreement found between the numerical and experimental results validates the new proposed system.

${ }^{1}$ D. Guicking and E. Lorenz, "An Active Sound Absorber with Porous Plate,' J. Vib. Acoust. Stress Reliab. Design 106, 389-392 (1984).

${ }^{2}$ D. Thenail, M. Galland, M. Sunyach, and M. Sunhack, "Active Enhancement of the Absorbent Properties of a Porous Material," Smart Mater. Struct. 3, 18-25 (1994)

${ }^{3}$ C. R. Fuller, M. J. Bronzel, C. H. Gentry, and D. E. Whittington, “'Control of Sound Radiation/Reflection with Adaptive Foams,' in Proceedings of Noise-Con 1994 Fort Lauderdale, Florida 1-4 May, pp. 429-436.

${ }^{4}$ S. Beyene, "Feasibility study of a Hybrid Passive/Active Noise Absorption System," Master's thesis, Virginia Polytechnic Institute and State University 1995.

${ }^{5}$ D. Guicking and K. Karcher, "Active Impedance Control for OneDimensional Sound,' J. Vib. Acoust. Stress Reliabil. Design 106, 393396 (1984).

${ }^{6}$ L. Beranek and I. L. Vèr, Noise and Vibration Control Engineering: Principles and Applications (Wiley, New York, 1992).

${ }^{7}$ C. D. Smith and T. L. Parrot, "Comparison of three methods for measuring acoustic properties of bulk materials," J. Acoust. Soc. Am. 74, 1577$1582(1983)$

${ }^{8}$ M. Mongy, "Acoustical Properties of Porous Materials," Acustica 28, 243-247 (1973)

9 "'Standard Test Method for Impedance and Absorption of Acoustical Materials by the Impedance Tube Method,' ASTM C384, Philadelphia, USA (1990).

${ }^{10}$ F. J. Fahy, "A Technique for Measuring Sound Intensity with a Sound Level Meter," Noise Control Eng. J. 9.(3), 155-162 (1977).

${ }^{11}$ B. Widrow and S. D. Stearns, Adaptive Signal Processing (Prentice-Hall, Englewood Cliffs, NJ, 1985). 